\title{
Overlap functions in correlation methods and quasifree nucleon knockout
}

\author{
from ${ }^{16} \mathrm{O}$ \\ M.K. Gaidarov, K.A. Pavlova, A.N. Antonov, M.V.Stoitsov, and S.S. Dimitrova \\ Institute of Nuclear Research and Nuclear Energy, Bulgarian Academy of Sciences, Sofia 1784, Bulgaria \\ C. Giusti \\ Dipartimento di Fisica Nucleare e Teorica, Università di Pavia, \\ Istituto Nazionale di Fisica Nucleare, Sezione di Pavia, Pavia, Italy \\ The cross sections of the $\left(e, e^{\prime} N\right)$ and $(\gamma, p)$ reactions on ${ }^{16} \mathrm{O}$ are calculated, for the \\ transitions to the $1 / 2^{-}$ground state and the first $3 / 2^{-}$excited state of the residual \\ nucleus, using single-particle overlap functions obtained on the basis of one-body den- \\ sity matrices within different correlation methods. The electron-induced one-nucleon \\ knockout reaction is treated within a nonrelativistic DWIA framework. The theoreti- \\ cal treatment of the $(\gamma, p)$ reaction includes both contributions of the direct knockout \\ mechanism and of meson-exchange currents. The results are sensitive to details of the \\ different overlap functions. The consistent analysis of the reaction cross sections and the \\ comparison with the experimental data make it possible to study the nucleon-nucleon \\ correlation effects.
}

\section{INTRODUCTION}

Quasifree $\left(e, e^{\prime} p\right)$ knockout reactions have proved to be a powerful tool for nuclear structure investigations. The large amount of data from these processes now available [1] gives detailed information on single-particle (s.p.) aspects of nuclear structure, revealing the properties of the nucleon-hole states contained in the hole spectral function. In this way, it is possible to point out the validity and limits of the s.p. description of nuclei.

The knowledge of the hole spectral function provides information on the spectroscopic factors for the removal process and on the momentum distribution of transitions to discrete final states of the residual nucleus. The spectroscopic factors obtained from the data exhibit a remarkable fragmentation over final states, thus indicating that nucleon-nucleon $(\mathrm{NN})$ correlations are not negligible [3,8,9]. The nucleon momentum distributions extracted for a variety of nuclei show unambiguosly the existence of high- 
momentum components [10] which is not the case in the mean-field approximation (MFA). They are caused by the short-range and tensor $\mathrm{NN}$ correlations in nuclei which originate from specific peculiarities of the nucleon-nucleon forces at small distances.

Along with the experimental studies, a precise theoretical treatment is also needed, taking correctly into account all main ingredients of the cross sections and regulating various approximations. Only in this way a proper comparison with experimental data is possible and reliable nuclear structure information can be extracted. The experimental momentum distributions are reproduced, with a good degree of accuracy, in a wide range of nuclei and in different kinematics, by a nonrelativistic treatment based on the distorted wave impulse approximation (DWIA), where also spin dependence in the final-state interactions (FSI) and Coulomb distortion of electron waves are taken into account [2,11]. Similar approaches based on a fully relativistic DWIA treatment are also available [12].

The exclusive nature of the quasifree knockout reactions in which the emitted nucleon is experimentally detected, is reflected theoretically in a transition amplitude which can be expressed in terms of the s.p. function representing the overlap between the target and residual nucleus states. In standard DWIA calculations phenomenological s.p. bound state wave functions are usually adopted, which do not include correlations. The normalization of the wave function, that is identified with the spectroscopic factor, is fitted to the data and its deviation from the predictions of the MFA is usually interpreted as evidence for the presence of correlations.

Explicit calculations of the hole spectral function and of fully correlated overlap functions for complex nuclei are very difficult. Only very recently the first successful parameter-free comparison of experiment and theory including the absolute normalization in $p$-shell nuclei has been performed for the ${ }^{7} \operatorname{Li}\left(e, e^{\prime} p\right)$ reaction [13]. For heavier nuclei, the effects of a spectral function containing short-range and tensor correlations on the ${ }^{16} \mathrm{O}\left(e, e^{\prime} p\right)$ reaction have been investigated in ref. [14]. The effects of a spectral function containing long-range correlations on the same reaction have been investigated in ref. [15]. In both cases a fair agreement with the shape of the experimental momentum distributions is obtained, but the size of the experimental cross section is overestimated and the spectroscopic factors determined by a fit to the data are thus lower than those predicted by the calculation of the spectral function. A calculation able to account for effects of both short-range (SRC) and long-range correlations (LRC) is extremely difficult, since it requires excessively large model space. A method to deal with SRC and LRC consistently has been proposed and applied to calculate the spectroscopic factors for one-nucleon 
knockout from ${ }^{16} \mathrm{O}[16]$.

It has been shown recently that absolute spectroscopic factors and overlap functions for one-nucleon removal reactions can be extracted from the one-body density matrix (OBDM) of the target nucleus [17]. The advantage of this procedure is that it avoids the complicated task of calculating the total nuclear spectral function. The procedure for extracting bound-state overlap functions has been applied [18 22] to OBDM emerging from various correlation methods such as the Jastrow correlation method (JCM) [23], the Correlated Basis Function (CBF) theory [19,24] and the Green function method (GFM) [25]. It has been shown that these functions are of particular importance, since they contain nucleon correlations which are accounted for to different extent in the various theoretical methods considered. On the other hand, their reliability for analyzing quantities which are sensitive to the NN correlations can be proved. The applicability of the theoretically calculated overlap functions has been tested in the description of the ${ }^{16} \mathrm{O}(p, d)$ pickup reaction [20 22] and of the ${ }^{40} \mathrm{Ca}(p, d)$ reaction (within the JCM) [20]. It has been found a good overall agreement between the calculated and the empirical cross sections. It has been pointed out also that acceptable spectroscopic factors can be obtained with the method proposed. Considering the role of the short-range and tensor correlations, it has been concluded that the LRC corresponding to collective degrees of freedom have to be taken also into account in order to achieve a better agreement with the $(p, d)$ data. The LRC can have sizable effects on the spectroscopic factors, on the shape of the overlap function and, as a consequence, on the cross sections.

In the present work the s.p. overlap functions obtained in different correlation methods mentioned above are used firstly to calculate the cross sections of the ${ }^{16} \mathrm{O}\left(e, e^{\prime} n\right)$ and ${ }^{16} \mathrm{O}\left(e, e^{\prime} p\right)$ knockout reactions. The aim of this investigation is to clarify the importance of the effects of various types of correlations on the reaction cross sections also in comparison with empirical $\left(e, e^{\prime} p\right)$ data. The calculation of the cross sections is based on the same nonrelativistic DWIA treatment 11] already used for the analysis of many experimental data. Secondly, the s.p. overlap functions are also used to calculate the cross section of the ${ }^{16} \mathrm{O}(\gamma, p)$ reaction. For the photon-induced reaction we have adopted the theoretical treatment of ref. [26], where the contributions of the direct knockout mechanism (DKO) and of meson-exchange currents (MEC) are evaluated consistently. The comparison of calculations, with consistent theoretical ingredients and constrained parameters, for the $\left(e, e^{\prime} p\right)$ and $(\gamma, p)$ cross sections can enable us to check, in comparison with data, the consistency of the theoretical description of the two reactions. Moreover, it allows us to investigate the behaviour of overlap functions from different correlation methods in a wide 
range of momenta, in particular at the large values that can be sampled in the $(\gamma, p)$ reaction and where correlation effects are expected to be more sizable.

The theoretical framework for the calculation of the $\left(e, e^{\prime} N\right)$ and $(\gamma, p)$ cross sections is presented in Section 2, together with the procedure to extract the s.p. overlap functions from the OBDM of the target nucleus. A short description of the correlation methods used is also given. The results of the calculations are presented and discussed in Section 3. The concluding remarks are given in Section 4.

\section{THEORETICAL APPROACH}

\section{A. The DWIA formalism for the $\left(e, e^{\prime} N\right)$ reaction}

The cross sections of the ${ }^{16} \mathrm{O}\left(e, e^{\prime} n\right)$ and ${ }^{16} \mathrm{O}\left(e, e^{\prime} p\right)$ reactions have been calculated with the code DWEEPY [11], which was able to give a good description of the $\left(e, e^{\prime} p\right)$ experimental momentum distributions, for transitions to different final states, in a wide range of nuclei and in different kinematics (see, e.g., ref. [2] and, more specifically for the analysis of the ${ }^{16} \mathrm{O}\left(e, e^{\prime} p\right)$ reaction, ref. «]). The calculation is based on a nonrelativistic DWIA description of the quasifree nucleon knockout process and accounts for both FSI and Coulomb distortion of the electron waves. The essential features of the formalism are given in this section. More details can be found in refs. [2,11].

In the one-photon exchange approximation, the general expression of the coincidence unpolarized cross section for the reaction induced by an electron, with momentum $\boldsymbol{p}_{0}$ and energy $E_{0}$, with $E_{0}=\left|\boldsymbol{p}_{0}\right|=p_{0}$, where a nucleon, with momentum $\boldsymbol{p}^{\prime}$ end energy $E^{\prime}$, is ejected from a nucleus, can be written in terms of four structure functions $W_{\mathrm{i}}$, as [2]

$$
\begin{array}{cc}
\frac{\mathrm{d}^{3} \sigma}{\mathrm{d} E_{0}^{\prime} \mathrm{d} \Omega_{0}^{\prime} \mathrm{d} \Omega^{\prime}}=\frac{\pi e^{2}}{2 q} \Gamma_{\mathrm{V}} \Omega_{\mathrm{f}} f_{\mathrm{rec}} \quad & {\left[\epsilon_{\mathrm{L}} W_{\mathrm{L}}+W_{\mathrm{T}}+\epsilon W_{\mathrm{TT}} \cos 2 \phi\right.} \\
\left.+\sqrt{\epsilon_{\mathrm{L}}(1+\epsilon)} W_{\mathrm{TL}} \cos \phi\right]
\end{array}
$$

where $e^{2} / 4 \pi \simeq 1 / 137, E_{0}^{\prime}$ is the energy of the scattered electron, with momentum $\boldsymbol{p}^{\prime}{ }_{0}$, and $\phi$ is the angle between the plane of the electrons and the plane containing the momentum transfer $\boldsymbol{q}$ and $\boldsymbol{p}^{\prime}$. The quantity

$$
\epsilon=\left(1-\frac{2 \boldsymbol{q}^{2}}{q_{\mu}^{2}} \tan ^{2} \frac{\theta}{2}\right)^{-1}
$$

measures the polarization of the virtual photon exchanged by the electron scattered at an angle $\theta$ and 


$$
\epsilon_{\mathrm{L}}=-\frac{q_{\mu}^{2}}{\boldsymbol{q}^{2}} \epsilon
$$

where $q_{\mu}^{2}=\omega^{2}-\boldsymbol{q}^{2}$, with $\omega=E_{0}-E_{0}^{\prime}$ and $\boldsymbol{q}=\boldsymbol{p}_{0}-\boldsymbol{p}_{0}^{\prime}$, is the four-momentum transfer. The factor

$$
\Gamma_{\mathrm{V}}=\frac{e^{2}}{8 \pi^{3}} \frac{E_{0}^{\prime}}{E_{0}} \frac{q}{q_{\mu}^{2}} \frac{1}{\epsilon-1}
$$

is the flux of virtual photons, $\Omega_{\mathrm{f}}=p^{\prime} E^{\prime}$ is the phase-space factor and

$$
f_{\mathrm{rec}}^{-1}=1-\frac{E^{\prime}}{E_{\mathrm{B}}} \frac{\boldsymbol{p}^{\prime} \cdot \boldsymbol{p}_{\mathrm{B}}}{\boldsymbol{p}^{\prime 2}}
$$

is the inverse of the recoil factor. The quantity $E_{\mathrm{B}}$ is the total relativistic energy of the residual nucleus with momentum $\boldsymbol{p}_{\mathrm{B}}=\boldsymbol{q}-\boldsymbol{p}^{\prime}$.

The structure functions represent the response of the nucleus to the longitudinal $(\mathrm{L})$ and transverse (T) components of the electromagnetic interaction. They are obtained from suitable combinations of the components of the hadron tensor [2] and are thus given by bilinear combinations of the Fourier transforms of the transition matrix elements of the nuclear charge-current density operator taken between initial and final nuclear states

$$
J^{\mu}(\boldsymbol{q})=\int\left\langle\Psi_{\mathrm{F}}\left|\hat{J}^{\mu}(\boldsymbol{r})\right| \Psi_{\mathrm{I}}\right\rangle \mathrm{e}^{\mathrm{i} \boldsymbol{q} \cdot \boldsymbol{r}} \mathrm{d} \boldsymbol{r}
$$

These integrals represent the basic ingredients of the calculation.

The DWIA treatment of the matrix elements in Eq. (6) is based on the following assumptions :

i) An exclusive process is considered, where the residual nucleus is left in a discrete eigenstate $\left|\Psi_{\alpha}^{\mathrm{B}}(E)\right\rangle$ of its Hamiltonian, with energy $E$ and quantum numbers $\alpha$.

ii) The final nuclear state is projected onto the channel subspace spanned by the vectors corresponding to a nucleon, at the position $\boldsymbol{r}_{1}$, and the residual nucleus in the state $\left|\Psi_{\alpha}^{\mathrm{B}}(E)\right\rangle$. This assumption is justified by the asymptotic configuration considered for the final state.

iii) The nuclear-current operator does not connect different channel subspaces. Thus, also the initial state is projected onto the selected channel subspace. This assumption is the basis of the direct knockout mechanism.

Then, the transition matrix elements in Eq. (6) can be written in a one-body representation as

$$
J^{\mu}(\boldsymbol{q})=\int \chi_{E \alpha}^{(-) *}\left(\boldsymbol{r}_{1}\right) \hat{J}_{\mathrm{eff}}^{\mu}\left(\boldsymbol{r}, \boldsymbol{r}_{1}\right) \phi_{E \alpha}\left(\boldsymbol{r}_{1}\right)\left[S_{\alpha}(E)\right]^{1 / 2} \mathrm{e}^{\mathrm{i} \boldsymbol{q} \cdot \boldsymbol{r}} \mathrm{d} \boldsymbol{r} \mathrm{d} \boldsymbol{r}_{1}
$$

where spin and isospin indices have been omitted for simplicity.

In Eq. (7) 


$$
\chi_{E \alpha}^{(-)}\left(\boldsymbol{r}_{1}\right)=\left\langle\Psi_{\alpha}^{\mathrm{B}}(E)\left|a\left(\boldsymbol{r}_{1}\right)\right| \Psi_{\mathrm{F}}\right\rangle
$$

where $a\left(\boldsymbol{r}_{1}\right)$ is an annihilation operator for a nucleon with coordinate $\boldsymbol{r}_{1}$, is the s.p. distorted wave function of the ejectile and the overlap function

$$
\left[S_{\alpha}(E)\right]^{1 / 2} \phi_{E \alpha}\left(\boldsymbol{r}_{1}\right)=\left\langle\Psi_{\alpha}^{\mathrm{B}}(E)\left|a\left(\boldsymbol{r}_{1}\right)\right| \Psi_{\mathrm{I}}\right\rangle
$$

describes the residual nucleus as a hole state in the target. The spectroscopic strength $S_{\alpha}(E)=\left\langle\phi_{E \alpha} \mid \phi_{E \alpha}\right\rangle$ is the norm of the overlap integral and gives the probability of removing from the target a nucleon at $\boldsymbol{r}_{1}$ leaving the residual nucleus in the state $\left|\Psi_{\alpha}^{\mathrm{B}}(E)\right\rangle$.

The scattering state in Eq. (8) and the normalized bound state $\phi_{E \alpha}\left(\boldsymbol{r}_{1}\right)$ in Eq. (9) are consistently derived from an energy-dependent optical model Feshbach Hamiltonian and, as such, they are not orthogonal. The use of the effective one-body operator $\hat{J}_{\text {eff }}^{\mu}$ in Eq. (7) removes the orthogonality defect of the model wave functions and takes into account space truncation effects [27]. The orthogonality defect is however negligible in the usual kinematics for the $\left(e, e^{\prime} p\right)$ reaction, and in actual DWIA calculations $\hat{J}_{\text {eff }}^{\mu}$ is generally replaced by the bare nuclear electromagnetic current operator. Two-body currents were also included in different theoretical approaches, but they do not give a relevant contribution to the calculated cross sections 28 . 30 .

In standard DWIA calculations the nucleon scattering state is eigenfunction of a phenomenological local spin-dependent optical potential, determined through a fit to elastic nucleon-nucleus scattering data including cross sections and polarizations. The nonlocality of the original Feshbach Hamiltonian is taken into account in the distorted wave function by means of the Perey factor [31].

In previous calculations phenomenological bound-state wave functions were usually adopted for the overlap functions. In the analysis of data these functions were calculated in a Woods-Saxon well, where the radius and the spectroscopic factor were considered as free parameters and were determined to reproduce the experimental momentum distributions, and the well depth was adjusted to reproduce the experimentally observed separation energy of the bound final state.

In this paper we have used overlap functions deduced from various correlation methods which do not contain any free parameters. A short explanation about how these overlap functions have been calculated on the basis of the OBDM and their main features are given in sec. 2.2.

The Coulomb distortion of the electron wave functions has been treated with a high-energy expansion in inverse powers of the electron energy [11]. For a light nucleus as ${ }^{16} \mathrm{O}$, however, an accurate description 
of Coulomb distortion is already given by the simple effective momentum approximation, where the momenta of incoming and outgoing electrons are changed into effective momenta [11].

Theoretical results and experimental data are usually presented, for specific values of the missing energy $E_{\mathrm{m}}=\omega-T^{\prime}-T_{\mathrm{B}}=E_{\mathrm{s}}+E_{\mathrm{x}}$, where $T^{\prime}$ and $T_{\mathrm{B}}$ are the kinetic energies of the outgoing nucleon and of the residual nucleus, respectively, $E_{\mathrm{s}}$ is the nucleon separation energy at threshold and $E_{\mathrm{x}}$ is the excitation energy of the residual nucleus, in terms of reduced cross sections, defined by

$$
\rho\left(p_{m}\right)=\int_{\Delta E_{\mathrm{m}}} \frac{\mathrm{d}^{3} \sigma}{\mathrm{d} E_{0}^{\prime} \mathrm{d} \Omega_{0}^{\prime} \mathrm{d} \Omega^{\prime}} \frac{1}{K \sigma_{\mathrm{eN}}} \mathrm{d} E_{\mathrm{m}},
$$

as a function of the missing momentum $p_{m}=\left|\boldsymbol{p}_{\mathrm{m}}\right|$, which is the magnitude of the recoil momentum of the residual nucleus $\boldsymbol{p}_{\mathrm{B}}$. In Eq. (10), $K=\Omega_{\mathrm{f}} f_{\text {rec }}$ and $\sigma_{\mathrm{eN}}$ is the elementary off-shell electron-nucleon scattering cross section. The CC1 prescription of ref. 32 is usually taken for $\sigma_{\mathrm{eN}}$.

Thus, the information contained in the differential cross section is reduced to a twofold function of $E_{\mathrm{m}}$ and $p_{\mathrm{m}}$. The integral in Eq. (10) is taken over the energy interval $\Delta E_{\mathrm{m}}$ that contains the peak of the transition under study. Calculations are usually performed for a single kinematics, corresponding to a central value of the phase-space volume in the region of the peak. Then, the reduced cross section in Eq. (10) can be written as [2]

$$
\rho^{\mathrm{th}}\left(p_{\mathrm{m}}\right)=S_{\alpha} n_{\alpha}\left(p_{\mathrm{m}}\right)
$$

where it is assumed that $n_{\alpha}\left(p_{\mathrm{m}}\right)$ is independent of the energy in the interval $\Delta E_{\mathrm{m}}$ and

$$
S_{\alpha}=\int_{\Delta E_{\mathrm{m}}} S_{\alpha}\left(E_{\mathrm{m}}\right) \mathrm{d} E_{\mathrm{m}}
$$

is the spectroscopic factor of the involved hole.

In the plane wave impulse approximation (PWIA), where FSI are neglected, $\boldsymbol{p}_{\mathrm{m}}$ is equal to the opposite of the initial momentum of the emitted nucleon in the nucleus, the cross section is factorized as

$$
\frac{\mathrm{d}^{3} \sigma}{\mathrm{d} E_{0}^{\prime} \mathrm{d} \Omega_{0}^{\prime} \mathrm{d} \Omega^{\prime}}=K \sigma_{\mathrm{eN}} S_{\alpha} \sum_{\alpha}\left|\phi_{\alpha}\left(\boldsymbol{p}_{\mathrm{m}}\right)\right|^{2}
$$

and the reduced cross section is the squared Fourier transform of the overlap function of Eq. (9).

In DWIA, the distortion of the electron and outgoing proton waves destroys the factorization, but it is still useful to define the reduced cross section of Eq. (10), that can be regarded as the nucleon momentum distribution modified by distortion and kinematics. This is the quantity that is presented is Section 3.1. 


\section{B. The overlap functions and their relationship with the one-body density matrix}

The quantities related to the $(A-1)$-particle system, such as the overlap functions, the separation energies and the spectroscopic factors for its bound states can be fully determined in principle by the one-body density matrix for the ground state of the $A$-particle system [17]. This unique relationship holds generally for quantum many-body systems with sufficiently short-range forces between the particles. Below we will give briefly how the overlap function can be obtained by using the asymptotic behaviour of the OBDM.

The overlap function is related to the hole spectral density function which takes part in the expression for the cross section of the exclusive $\left(e, e^{\prime} N\right)$ reaction. When the final nuclear state is undetermined, the integral of the spectral density function over the energy spectrum gives the one-body density matrix $\rho\left(\boldsymbol{r}, \boldsymbol{r}^{\prime}\right)$. The latter can be expressed in terms of the overlap functions (9) in the form:

$$
\rho\left(\boldsymbol{r}, \boldsymbol{r}^{\prime}\right)=\sum_{\alpha} S_{\alpha} \phi_{\alpha}^{*}(\boldsymbol{r}) \phi_{\alpha}\left(\boldsymbol{r}^{\prime}\right)
$$

In the case of a target nucleus with $J^{\pi}=0^{+}$, each eigenstate of the residual nucleus is characterized by the quantum numbers $l j$, i.e., $\alpha \equiv n l j$, with $n$ being the number of the state with given $l$ and $j$. It is known 33 that the overlap functions associated with the bound states of the $(A-1)$ - and $(A+1)$-nucleon systems are eigenstates of a s.p. Schrödinger equation in which the mass operator plays the role of a potential. Due to its finite range, the asymptotic behaviour of the radial part of the neutron overlap functions for the bound states of the $(A-1)$-system is given by [17, 34, 35]:

$$
\phi_{n l j}(r) \rightarrow C_{n l j} \exp \left(-k_{n l j} r\right) / r
$$

where

$$
k_{n l j}=\frac{1}{\hbar}\left[2 m_{\mathrm{n}}\left(E_{n l j}^{A-1}-E_{0}^{A}\right)\right]^{1 / 2} .
$$

In Eq.(16) $m_{\mathrm{n}}$ is the neutron mass, $E_{0}^{A}$ is the ground state energy of the target $A$-nucleus and $E_{n l j}^{A-1}$ is the energy of the $n l j$-state of the $(A-1)$-nucleus. For protons some mathematical complications arise due to an additional long-range part originating from the Coulomb interaction, but the general conclusions of the consideration remain the same. The asymptotic behaviour of the radial part of the corresponding proton overlap functions reads

$$
\phi_{n l j}(r) \rightarrow C_{n l j} \exp \left[-k_{n l j} r-\eta \ln \left(2 k_{n l j} r\right)\right] / r
$$


where $\eta$ is the Coulomb (or Sommerfeld) parameter and $k_{n l j}$ (16) contains the mass of the proton.

The lowest $\left(n_{0} l j\right)$ bound state overlap function (for neutrons) is determined by the asymptotic behaviour of the corresponding partial radial contribution of the one-body density matrix $\rho_{l j}\left(r, r^{\prime}\right)$ $\left(r^{\prime}=a \rightarrow \infty\right):$

$$
\phi_{n_{0} l j}(r)=\frac{\rho_{l j}(r, a)}{C_{n_{0} l j} \exp \left(-k_{n_{0} l j} a\right) / a},
$$

where the constants $C_{n_{0} l j}$ and $k_{n_{0} l j}$ are completely determined by $\rho_{l j}(a, a)$. In this way the separation energy

$$
\epsilon_{n_{0} l j} \equiv E_{n_{0} l j}^{A-1}-E_{0}^{A}=\frac{\hbar^{2} k_{n_{0} l j}^{2}}{2 m_{\mathrm{n}}}
$$

and the spectroscopic factor $S_{n_{0} l j}=\left\langle\phi_{n_{0} l j} \mid \phi_{n_{0} l j}\right\rangle$ can be determined as well. As shown in ref. [17], the procedure also yields in principle all bound state overlap functions with the same multipolarity, if they exist. For instance, the overlap function for the next bound state is:

$$
\phi_{n_{1} l j}(r)=\frac{\rho_{l j}(r, a)-\phi_{n_{0} l j}(r) \phi_{n_{0} l j}(a)}{C_{n_{1} l j} \exp \left(-k_{n_{1} l j} a\right) / a} .
$$

The applicability of this theoretical scheme has been demonstrated in refs. [18, 19, 36]. A simple but effective approach accounting for the SRC within the Jastrow correlation method in its low-order approximation has been used to obtain the OBDM [23] and to calculate the s.p. overlap functions [18]. Another type of overlap functions has been obtained [19] within the framework of the CBF theory using the cluster expansion and correlation functions from Variational Monte Carlo calculations with the Argonne potential. In refs. 21.22] calculations of overlap functions corresponding to the OBDM 24 obtained within a model treating correlations independent on the isospin up to the first order in the cluster expansion and using the Fermi Hypernetted Chain Technique have been performed. Finally, overlap functions have been obtained [21,22] on the basis of the realistic OBDM constructed within the GFM [25]. Using the systematic analysis of s.p. overlap functions obtained from realistic density matrices just mentioned one can distinguish between the effects of different types of correlations on quantities such as overlap functions and spectroscopic factors of quasihole states. In the present paper all these overlap functions are explored for the analysis of the quasifree nucleon knockout from the ${ }^{16} \mathrm{O}$ nucleus.

It has been shown in refs. [21]22] that due to the inclusion of short-range as well as tensor correlations the overlap functions are peaked at smaller distance in the interior region of the nucleus in comparison with the Hartree-Fock wave functions. In the momentum space this leads to a slight redistribution of the 
strength from the low- to the high-momentum region. Considering the role of both central and tensor correlations it is found that the correlation effects on the spectroscopic factors of the hole states are dominated by the tensor channel of the interaction.

The s.p. overlap functions have been used also as form factors for the description of ${ }^{16} \mathrm{O}(p, d)$ 20 22 and ${ }^{40} \mathrm{Ca}(p, d)$ [20] pickup reactions. It was shown, as an important result from the analysis, that it is not necessary the theoretically obtained angular distributions to be normalized by means of spectroscopic factors because the latter are already included in the overlap functions. Thus having the procedure for calculating such important quantities as the overlap functions and the spectroscopic factors it is desirable to apply them also for a consistent study of one-nucleon knockout reactions induced by electrons and photons, which is the aim of the present paper.

\section{The theoretical framework for the $(\gamma, p)$ reaction}

The differential cross section for the reaction induced by a photon, with energy $E_{\gamma}$, where a nucleon, with momentum $\boldsymbol{p}^{\prime}$, is ejected from a nucleus, can be written as [2]

$$
\frac{\mathrm{d} \sigma}{\mathrm{d} \Omega^{\prime}}=\frac{\pi e^{2}}{2 E_{\gamma}} \Omega_{\mathrm{f}} f_{\mathrm{rec}} W_{\mathrm{T}}
$$

where $\Omega_{\mathrm{f}}, f_{\text {rec }}$ and $W_{\mathrm{T}}$ have the same expressions as in Eq. (11). In contrast to the case of the electroninduced reaction, where both longitudinal and transverse components of the nuclear response contribute, here only the pure transverse response function $W_{\mathrm{T}}$ occurs.

The DWIA treatment presented in Section 2.1 can be applied also to $(\gamma, N)$ reactions. Photon-induced nucleon emission appears of particular interest for our purposes, since the cross sections are expected to be very sensitive to details of the overlap functions and to effects of SRC. In fact, in this case, $\omega=|\boldsymbol{q}|=E_{\gamma}$, and the mismatch between the momentum transfer and the momentum of the outgoing nucleon is quite large. Thus, if the reaction proceeds through a DKO mechanism, only the high-momentum components of the nuclear wave function are probed. On the other hand, the validity of the DKO mechanism, which is clearly stated for $\left(e, e^{\prime} p\right)$, is much more questionable for $(\gamma, N)$ reactions, where two-nucleon processes, such as those involving meson-exchange currents are expected to be important or even dominant. They are certainly dominant in the $(\gamma, n)$ reaction, where the DKO mechanism, even in its most sophisticated

version [37, gives but a small fraction of the experimental cross sections, while for the $(\gamma, p)$ reaction the contribution of DKO is much more relevant. Nonrelativistic DWIA calculations, based on the same 
approach presented in Section 2.1 for the $\left(e, e^{\prime} N\right)$ reaction [38], and more recent relativistic calculations, also based on the DKO mechanism [39], are able to give a fair description of data. The results, however, are very sensitive to the theoretical ingredients adopted for bound and scattering states. On the other hand, various calculations in different theoretical approaches indicate that MEC play a prominent role also in the $(\gamma, p)$ reaction 26,40 .

Therefore in this paper the cross sections of the exclusive $(\gamma, p)$ reaction have been calculated in the theoretical framework of ref. [26], where photoabsorption occurs, through one-body and two-body currents, on a pair of correlated nucleons: only one of them is then emitted, while the other one is reabsorbed in the residual nucleus. In this model MEC are included in the framework of the DKO model with FSI and thus the size and the relative weight of DKO and MEC can be evaluated consistently.

In the calculations of ref. [26] the correlated wave function of the pair was given by the product of shell-model s.p. bound state wave functions and of a central Jastrow type correlation function, which takes into account SRC. Here we have adopted one-nucleon overlap functions obtained, as it is explained in Section 2.2, from different realistic one-body density matrices and that already include SRC. The calculated cross section is thus the sum of two terms: the direct contribution of the one-body current, which corresponds to the quasifree DKO considered in Section 2.1, and the exchange contribution of the two-body current. In this model MEC are explicitly taken into account in a microscopic and unfactorized calculation. Moreover, a consistent analysis, with consistent ingredients, i.e. overlap functions, spectroscopic factors and optical model parameters, can be performed of $\left(e, e^{\prime} p\right)$ and $(\gamma, p)$ reaction cross sections.

In order to reduce the complexity of the calculation, we have adopted the same approximations as in ref. [26]. Only the contribution of the two-body current due to the seagull diagrams has been included. Currents due to the pion-in-flight diagrams give a pure contribution much smaller than that due to the seagull diagrams and currents corresponding to diagrams with intermediate $\Delta$ isobar configurations become important only for photon energies above the pion-production threshold. Thus, the seagull current, here considered, should give the main contribution of the two-body current in the photon-energy range above the giant resonance and below the pion production threshold.

The spin-orbit part of the optical potential has been neglected in the calculations of the $(\gamma, p)$ cross section, as well as various effects considered in ref. [37] in the framework of the DKO model: chargeexchange FSI, orthogonality between initial and final states, antisymmetrization of the outgoing particle 
and recoil terms. These effects cannot be simply applied to the present approach, where also two-body currents are included. Their evaluation would require a specific and consistent treatment. In ref. [37] the sum of these effects turns out to be very important for $(\gamma, n)$, while for $(\gamma, p)$ it gives a much smaller contribution, which should be further reduced when also the two-body current is added in the calculated cross sections.

Thus, although with some approximations, the present treatment should include all the most important and essential ingredients contributing to the cross section of the $(\gamma, p)$ reaction in the photon-energy range between 50 and $100 \mathrm{MeV}$.

\section{RESULTS AND DISCUSSION}

\section{A. The ${ }^{16} \mathbf{O}\left(e, e^{\prime} n\right)$ and ${ }^{16} \mathbf{O}\left(e, e^{\prime} p\right)$ reactions}

In this section we discuss results for the reduced cross sections of the ${ }^{16} \mathrm{O}\left(e, e^{\prime} n\right)$ and ${ }^{16} \mathrm{O}\left(e, e^{\prime} p\right)$ reactions. In PWIA, where the effects of the FSI between the outgoing nucleon and the residual $(A-1)$ nuclear system are neglected, the reduced cross section $\rho\left(p_{\mathrm{m}}\right)$, defined in Eq. (11), is the squared Fourier transform of the overlap funtion. In DWIA it can be regarded as the nucleon momentum distribution modified by distortion and kinematics.

In standard DWIA calculations the overlap function is generally replaced by a phenomenological s.p. bound-state wave function which is eigenfunction of a mean-field Woods-Saxon potential. In the analysis of the $\left(e, e^{\prime} p\right)$ data the well depth is determined to reproduce the separation energy values and the radius is adjusted to fit the shape of the momentum distribution. In the present work we have used overlap functions derived from different calculations of the OBDM. We would like to emphasize that this is the correct theoretical procedure which in principle has to be used for an accurate description of $\left(e, e^{\prime} N\right)$ knockout reactions. Our calculations are performed by using the s.p. overlap functions obtained from Eqs. (15) and (17) for the neutron and proton bound states, respectively. The results presented for both $\left(e, e^{\prime} n\right)$ and $\left(e, e^{\prime} p\right)$ reactions are obtained using the neutron overlap functions. The application of the latter to the $\left(e, e^{\prime} p\right)$ reaction can be justified by the fact that the proton and neutron overlap functions are very similar and the use of the correct proton one leads to almost identical results in this case.

Our analysis is made for the transitions to the $1 / 2^{-}$ground state and to the first $3 / 2^{-}$excited state of the residual nucleus (at excitation energy $E_{\mathrm{x}}=6.18 \mathrm{MeV}$ for ${ }^{15} \mathrm{O}$ in the case of the $\left(e, e^{\prime} n\right)$ reaction and 
at $E_{\mathrm{x}}=6.3 \mathrm{MeV}$ for ${ }^{15} \mathrm{~N}$ in the case of the $\left(e, e^{\prime} p\right)$ reaction), representing a knockout from the valence $1 p$ shell of ${ }^{16} \mathrm{O}$. Unfortunately, experiments on the $\left(e, e^{\prime} n\right)$ reactions have not been carried out so far, due to the difficulties in performing neutron detection in a coincidence reaction and, therefore, our results are theoretical predictions. In contrast, a large number of experiments have been performed over the past years on the $\left(e, e^{\prime} p\right)$ reactions [1] [7. We compare here our theoretical results for the ${ }^{16} \mathrm{O}\left(e, e^{\prime} p\right)$ reaction with the data taken at NIKHEF [4] in the so-called parallel kinematics. In this kinematics the momentum of the outgoing nucleon is fixed and is taken parallel or antiparallel to the momentum transfer. Different values of the missing momentum are obtained by varying the electron scattering angle and therefore the magnitude of the momentum transfer. This kinematics, that has been considered in most of the $\left(e, e^{\prime} p\right)$ experiments, represents a suitable example for the present investigation of the effects of different overlap functions in quasifree nucleon knockout reactions from ${ }^{16} \mathrm{O}$.

The reduced cross sections for the ${ }^{16} \mathrm{O}\left(e, e^{\prime} n\right)$ reaction as a function of the missing momentum and for the transitions to the $1 / 2^{-}$ground state and the $3 / 2^{-}$excited state of ${ }^{15} \mathrm{O}$ are displayed in Fig. 1 . The results obtained with different overlap functions are compared with those given by the Hartree-Fock (HF) wave function, which is calculated in a self-consistent way using the Skyrme-III interaction. Besides the HF wave function, whose norm is equal to one, all the overlap functions contain a spectroscopic factor. These factors are listed in Table I (column I) and were discussed in details in refs. [21,22]. They account for the contribution of correlations included in the OBDM which cause a depletion of the quasihole states. Only short-range central correlations are included in the OBDM of refs. 18,24], whereas also tensor correlations are taken into account in refs. 19,25. It was found that correlation effects on the spectroscopic factor of the hole states are dominated by the tensor channel of the interaction [21.22]. Indeed the spectroscopic factors in Table I are lower for the overlap functions including also tensor correlations. These overlap functions, however, do not include LRC, which should produce further depletion of the quasihole states [15, 16].

The reduced cross sections in Fig. 1 are sensitive to the shape of the various overlap functions used. The differences are considerable at large values of $p_{\mathrm{m}}$, where the cross section is several orders of magnitude lower than in the maximum region. Similar results for the different overlap functions as a function of $p_{\mathrm{m}}$ have been obtained also for the $\left(e, e^{\prime} p\right)$ reaction and in different kinematics. The deviations of the various results at large values of $p_{\mathrm{m}}$ are related to different accounting for the short-range $\mathrm{NN}$ correlations within the correlation methods used. SRC are particularly important in one-nucleon emission at large 
missing momenta and energy [14,41]. At high missing energies, however, other competing processes are also present and a clear identification of SRC can better be made by means of two-nucleon knockout reactions [42]. At low missing-energy values measurements over an extended range of missing momenta, in particular at large values, where the SRC effects seem to be more sizable, can test the various s.p. overlap functions and NN correlations.

The reduced cross sections for the ${ }^{16} \mathrm{O}\left(e, e^{\prime} p\right)$ reaction are shown in Fig. 2 in comparison with data. In order to reproduce the size of the experimental cross section a reduction factor has been applied to the theoretical results. These factors, which have been obtained by a fit of the calculated reduced cross sections to the data over the whole missing-momentum range considered in the experiment, are also listed in Table I (column II). In general, a good agreement with the shape of the experimental distribution is achieved. The results, however, are also sensitive to details of the various overlap functions. The best agreement with the data, for both transitions, is obtained with the overlap functions 2122 emerging from the OBDM calculated within the Green function method [25]. This is due to the substantial realistic inclusion of short-range as well as tensor correlations in the OBDM. The calculations based on the Green function theory [21,22] have shown that about $10 \%$ of the $1 p$ strength is removed by these correlations. The reduced cross section obtained by using the overlap function from ref. 19] gives also a good agreement for the $1 / 2^{-}$state, but a less satisfactory description of data for $3 / 2^{-}$. In contrast, the overlap function from ref. [18] gives a better description of the experimental distribution for the $3 / 2^{-}$than for the ground state. The shape of the experimental reduced cross sections can adequately be described also by the $\mathrm{HF}$ wave functions, in particular for $p_{\mathrm{m}} \leq 150 \mathrm{MeV} / c$. Only the overlap function extracted from the OBDM of ref. 24] is unable to give an adequate description of the experimental momentum distributions of the $1 / 2^{-}$state, while it gives a better agreement for the $3 / 2^{-}$state. In general, the agreement of the calculated reduced cross sections with data is somewhat better for the $3 / 2^{-}$than for the $1 / 2^{-}$state.

We note that even though a fair agreement with the shape of the experimental distributions is generally obtained in the present calculations, this agreement is not as good as in the analysis of ref. [田. The calculations have been performed in both cases with the same DWIA treatment and with the same optical potential [43], but in ref. [4] a s.p. phenomenological wave function was adopted, with some parameters adjusted to the data. In the present work overlap functions obtained within different correlation methods, which contain approximations but no free parameters have been used.

Only a reduction factor has been applied to the calculated cross sections to reproduce the data. The 
fact that our results overestimate the data may be explained on a theoretical basis by the observation that our overlap functions are deduced from calculations including only SRC but not LRC. The reduction factor can thus be considered as a further spectroscopic factor reflecting the depletion of the quasihole state produced by LRC. Of course, the discrepancy with the data can be due also to other effects not included or not adequately described by the theoretical treatment. For instance, a relativistic optical potential increases by about $15 \%$ the absorption due to FSI and thus gives a reduction of the calculated cross sections 12,44. On the other hand, a proper treatment of the center-of-mass motion leads to an enhancement of the spectroscopic factor by about 7\% 445. Also two-body currents may lead to small variations of the size of the calculated cross sections [29]. We note, however, that the reduction factors applied here to the calculated cross sections are not the result of a precise theoretical calculation. They have been obtained by a fit to the data and have only an indicative meaning. Small variations within 10-15\% around their values would not significantly change the comparison with data. In any case the reduction factors should mostly be ascribed to LRC, but for the HF wave function, which does not contain any kind of correlations. For this wave function the reduction factor accounts for both LRC and SRC. It is interesting to note that in the calculations with all the correlated overlap functions the reduction factors for the $1 p_{1 / 2}$ state turn out to be close to the spectroscopic factor (0.83) obtained in the theoretical approach of ref. 15 where only LRC are included. For $1 p_{3 / 2}$, however, the reduction factors are lower than the spectroscopic factor (0.85) calculated in the same approach.

In Table I we give, in addition, in column III the factor obtained by the product of the two factors in columns I and II. This factor can be considered as a total spectroscopic factor and can be attributed to the combined effect of SRC and LRC. Indeed for $1 p_{1 / 2}$ these factors are in reasonable agreement with the spectroscopic factor (0.76) calculated in ref. [16], where both SRC and LRC are consistently included. Also the HF wave function gives a total spectroscopic factor in agreement with the result of ref. 16] and a reasonable description of the shape of the experimental distribution in Fig. 2. This means that in the missing-momentum range considered by the experiment, the correlation effects are overwhelmed by the dominant quasihole component already present in the HF approximation. For the transition to the $1 / 2^{-}$ state a quite large value of the total spectroscopic factor is obtained with the overlap function extracted from the OBDM by adopting the Average Correlation Approximation [24]. This is due to the fact that in this approach the correlations are mainly produced by the central short-range components of the NN interaction. Moreover, this function is unable to reproduce correctly the shape of the experimental 
distribution.

The total spectroscopic factors obtained for the $3 / 2^{-}$state are lower than those calculated in ref. [16], but for the overlap function from ref. [19], which, on the other hand, gives for this state a worse description of the data. We note that also other analyses of the same data [4, 14, 15] gave for $3 / 2^{-}$a spectroscopic factor lower than for the ground state. It was noticed in ref. [14] that three $3 / 2^{-}$states are observed in ${ }^{15} \mathrm{~N}$ at low excitation energies and that LRC yield a splitting such that $86 \%$ of the total strength going to these states is contained in the data. This splitting is not observed in the calculations. If the total spectroscopic factors in Table I are divided by 0.86 to account for the splitting of the experimental strength, we obtain a value closer to that obtained for the $1 / 2^{-}$state and in the calculation of ref [16].

In the DWIA analysis of the data in ref. 四 phenomenological Woods-Saxon bound-state wave functions gave the "experimental" spectroscopic factors of 0.61 for $1 / 2^{-}$and 0.53 for $3 / 2^{-}$, somewhat lower than in the present work with theoretically calculated overlap functions.

The overlap functions here considered only include SRC and some of them also tensor correlations. It would be of great interest to avail theoretically calculated overlap functions where both SRC and LRC are consistently included and to investigate their effects on the size and the shape of the calculated cross sections and in comparison with data.

\section{B. The ${ }^{16} \mathbf{O}(\gamma, p)$ reaction}

In this section we present results of calculations for the ${ }^{16} \mathrm{O}(\gamma, p)$ reaction and discuss them in comparison with data. Calculations have been performed within the theoretical framework of ref. [26], where one-body and two-body currents are included and both contributions of DKO and MEC can be evaluated consistently. The same theoretical ingredients, i.e. s.p. overlap functions, spectroscopic factors and consistent optical potentials, have been adopted as in the calculations of the $\left(e, e^{\prime} p\right)$ cross section. Moreover, the reduction factor determined in comparison with the $\left(e, e^{\prime} p\right)$ data has been applied also in the comparison of the calculated $(\gamma, p)$ cross section with data. Since $(\gamma, p)$ calculations are extremely sensitive to the theoretical ingredients adopted for bound and scattering states, the use of constrained parameters should allow us to reduce ambiguities in the interpretation of the results and to perform a consistent study of the ${ }^{16} \mathrm{O}\left(e, e^{\prime} p\right)$ and ${ }^{16} \mathrm{O}(\gamma, p)$ reactions.

The aim of our investigation is twofold. On the one hand, we intend to check the consistency of the theoretical treatment for the two reactions. On the other hand, we want to investigate the sensitivity 
of the results to the various overlap functions and to NN correlations, which are included in the overlap functions within different theoretical frameworks, at large values of the missing momentum, where SRC effects are more sizable.

We restrict our analysis to photon-energy values where our theoretical treatment appears more reliable. Therefore we have performed calculations at $E_{\gamma}=60$ and $72 \mathrm{MeV}$, where ${ }^{16} \mathrm{O}(\gamma, p)$ data are available for the transition to the $1 / 2^{-}$ground state [46 48] and to the $3 / 2^{-}$excited state at $6.3 \mathrm{MeV}$ 48]. At these photon-energy values it is possible to sample in comparison with data $p_{\mathrm{m}}$ values between 250 and $400 \mathrm{MeV} / c$.

The angular distribution of the ${ }^{16} \mathrm{O}(\gamma, p){ }^{15} \mathrm{~N}_{\text {g.s. }}$ reaction at $E_{\gamma}=60 \mathrm{MeV}$ is displayed in Fig. 3. In the figure the results given by the sum of the one-body and of the two-body seagull currents are compared with the contribution given by the one-body current, which roughly corresponds to the DWIA treatment based on the DKO mechanism.

The DWIA calculations with different overlap functions exhibit considerable differences, in particular at backward angles, where larger values of $p_{\mathrm{m}}$ are probed. These differences are somewhat reduced when the two-body seagull current is added, but remain anyhow quite large.

The contribution of the one-body current represents a large part of the measured cross section, but none of the overlap functions used is able to give a proper description of the data. All the curves in DWIA lie well below the data, but that obtained with the overlap function from the OBDM of ref. [24], which is anyhow able to reproduce the size of the experimental cross section only at the lowest angles. A much better agreement with $(\gamma, p)$ data is obtained when MEC are added to the DWIA result. The HF wave function and the overlap function obtained from the OBDM of ref. [18] are able to reproduce the size of the experimental cross section, but only at low values of the outgoing proton angle. Thus they are unable to reproduce the shape of the distribution. The result with the overlap function from the OBDM of ref. 24] largely overshoots the data. Much better agreement with the shape of the experimental distribution is given by the other correlated overlap functions. We notice that a better overlap with the $(\gamma, p)$ data is obtained using the overlap function from the OBDM of ref. [25] and, to a lesser extent, also by that from ref. [19]. These overlap functions, obtained from calculations where short-range as well as tensor correlations are included, are also able to give the best agreement with $\left(e, e^{\prime} p\right)$ data for the $1 / 2^{-}$state in Fig. 2. In Fig. 3 calculations with these wave functions lie a bit below the data in the maximum region, but they give a fair agreement with the shape of the experimental distribution, in 
particular for the overlap function from ref. [25]. The existing discrepancies are anyhow not large and might be explained within the approximations of the theoretical model, or also by a bit lower reduction factor. We already observed in the $\left(e, e^{\prime} p\right)$ analysis of Section 3.1 that small variations around the values listed in Table I would not change significantly the comparison with $\left(e, e^{\prime} p\right)$ data, but would here improve the agreement with the experimental results for the $(\gamma, p)$ reaction, which is much more sensitive to the various theoretical ingredients. The fact that the overlap function from the OBDM of ref. 25] is able to give the best description of both $\left(e, e^{\prime} p\right)$ and $(\gamma, p)$ data is a strong indication in favour of a consistent analysis of the two reactions. Similar results are obtained in Fig. 4, where the angular distribution of the ${ }^{16} \mathrm{O}(\gamma, p){ }^{15} \mathrm{~N}_{\text {g.s. }}$ reaction is displayed at $E_{\gamma}=72 \mathrm{MeV}$. The results confirm the important role played by MEC to describe the size and the shape of the experimental cross section and the great sensitivity to the shape of the overlap function and to correlation effects. The behaviour of the angular distribution in comparison with data is similar to that of Fig. 3, at $E_{\gamma}=60 \mathrm{MeV}$. Also at $E_{\gamma}=72 \mathrm{MeV}$ the HF wave function and the overlap function from the OBDM of ref. [18] can reproduce the size of the experimental cross section only at low values of the scattering angle and are unable to give a proper description of the shape of the angular distribution. The cross section calculated with the overlap function from ref. [24] overshoots the data, while a fair agreement with data is obtained with the overlap function from ref. [25]. The cross section calculated with this overlap function as well as that calculated with the overlap function from ref. [19] are a bit higher than the data at low values of the outgoing proton angle, but the discrepancy is about the same as at $E_{\gamma}=60 \mathrm{MeV}$ and might be explained within the uncertainties of the theoretical treatment.

An example for the transition to the $3 / 2^{-}$state at $6.3 \mathrm{MeV}$ is presented in Fig. 5 , where the angular distribution of the ${ }^{16} \mathrm{O}(\gamma, p)$ reaction at $E_{\gamma}=72 \mathrm{MeV}$ is displayed in comparison with data. Similar results have been obtained at $E_{\gamma}=60 \mathrm{MeV}$. The results for this transition confirm the sensitivity to the overlap function and the important role of MEC in the cross section of the $(\gamma, p)$ reaction. However, the conclusions about comparison with data are in this case less clear. The size of the experimental cross section is already described by DWIA calculations, but with the overlap function derived from the OBDM of ref. 18. In contrast, the shape is much better reproduced by the more complete calculations including also the seagull current. On the other hand, these results including both one-body and two-body currents overshoot the data, but with the overlap function from ref. [18]. The overlap function from ref. [25], which is able to give the best agreement with $\left(e, e^{\prime} p\right)$ data also for this transition, is able to reproduce very 
well the shape of experimental angular distribution in Fig. 5, but the calculated cross section overshoots the data by a factor of about two. Only the overlap function from the OBDM of ref. [18] gives a fair agreement with the size and the shape of the $(\gamma, p)$ data in Fig. 5. The small discrepancy might be explained within the approximations of the model. For instance, an enhancement of the cross section at high values of the scattering angle should be given by the spin-orbit part of the optical potential [38], which has been neglected in the present approach. We want to remind that the overlap function from ref. [18] is also able to give in Fig. 2 a very good description of the $\left(e, e^{\prime} p\right)$ experimental reduced cross section for the transition to the same $3 / 2^{-}$state.

The results for the $3 / 2^{-}$state, although less clear than for the ground state of ${ }^{15} \mathrm{~N}$, can thus be considered as further evidence in favour of a consistent description, with the same theoretical ingredients, of $\left(e, e^{\prime} p\right)$ and $(\gamma, p)$ data. However, in order to draw definite conclusions, a more refined theoretical treatment of the $(\gamma, p)$ reaction is needed, where the approximations of the present approach are improved and a more careful comparison with data in a wider photon-energy range can be performed.

\section{SUMMARY AND CONCLUSIONS}

Single-particle overlap functions calculated, for the ${ }^{16} \mathrm{O}$ nucleus, on the basis of the OBDM emerging from various correlations methods have been used to calculate the cross sections of the $\left(e, e^{\prime} n\right),\left(e, e^{\prime} p\right)$ and $(\gamma, p)$ reactions, for the transitions to the $1 / 2^{-}$ground state and the first $3 / 2^{-}$excited state of the residual nucleus. These overlap functions contain short-range central and tensor correlations and include the spectroscopic factor. The aim of the present investigation was to clarify the importance of various types of correlations, which are accounted for to different extent in the theoretical methods considered, on the reaction cross sections and in comparison with data.

The reduced cross sections of the ${ }^{16} \mathrm{O}\left(e, e^{\prime} n\right)$ and ${ }^{16} \mathrm{O}\left(e, e^{\prime} p\right)$ knockout reactions have been calculated with the same nonrelativistic DWIA treatment which was successfully applied previously to the analysis of many $\left(e, e^{\prime} p\right)$ data. In the standard DWIA approach, however, phenomenological s.p. wave functions were used, with some parameters fitted to the data. In this paper the results have been obtained with theoretically calculated overlap functions which do not include free parameters.

The reduced cross sections are sensitive to the shape of the various overlap functions and exhibit considerable differences at large values of the missing momentum, where correlation effects are more sizable. The theoretical results are generally able to reproduce, with a fair agreement, the shape of the 
experimental reduced cross sections. The quality of the agreement, however, is sensitive to details of the different overlap functions.

In order to reproduce the size of the experimental data a reduction factor must be applied to the calculated reduced cross sections. This factor, that is extracted from a fit to the data, can be considered as a further spectroscopic factor to be mostly ascribed to LRC, which also cause a depletion of the quasihole states and which are not included in the overlap functions considered here. The spectroscopic factors accounting for SRC and LRC obtained in the present analysis are in reasonable agreement with those given by previous theoretical investigations.

Since both SRC and LRC have sizable effects on the spectroscopic factors, on the shape of the overlap function and, as a consequence, on the cross sections, a calculation of fully correlated overlap functions consistently including SRC and LRC, although extremely difficult, would be highly desirable and would allow a direct and parameter-free comparison with data.

The behaviour of the different overlap functions at high values of momenta and NN correlation effects can better be investigated in the $(\gamma, p)$ reaction. The cross section of the ${ }^{16} \mathrm{O}(\gamma, p)$ reaction has been calculated at $E_{\gamma}=60$ and $72 \mathrm{MeV}$. Consistent theoretical ingredients and the same spectroscopic factors extracted from the analysis of the ${ }^{16} \mathrm{O}\left(e, e^{\prime} p\right)$ reaction have constrained the calculations. The theoretical treatment of the photon induced reaction includes both contributions of the DKO mechanism and of the two-body pion seagull current.

The various overlap functions give considerable differences on the size and shape of the calculated cross sections. The contribution of the DKO mechanism is unable to describe the experimental data. The numerical results generally fall short of the data and all the curves are unable to reproduce the shape of the experimental angular distributions. The contribution of MEC is large. It significantly affects both the size and shape of the cross section and generally brings the calculated cross section in much better agreement with the data.

For the transition to the ground state of ${ }^{15} \mathrm{~N}$ the best and a fair description of the data is given by the overlap function able to give also the best description of the $\left(e, e^{\prime} p\right)$ data. This is a strong indication in favour of the consistency in the analysis of the $\left(e, e^{\prime} p\right)$ and $(\gamma, p)$ reactions. This result is partly confirmed also for the transition to the $3 / 2^{-}$excited state. In this case, however, the situation is less clear and larger discrepancies in comparison with data are obtained. These discrepancies are anyhow not too large and might be explained within the approximations of the model. 
A more refined theoretical treatment, which should consistently include SRC and LRC, as well as orthogonality, antisymmetry and c.m. effects, together with a more complete evaluation of two-body currents, would allow a more careful comparison with data in a wider kinematical range and would be needed to draw definite conclusions.

\section{ACKNOWLEDGMENTS}

One of us (C.G.) wishes to thank F.D. Pacati and M. Radici for useful discussions. The authors thank H. Müther, G. Co' and D. Van Neck for providing us the results for the OBDM used. The work is partly supported by the Bulgarian National Science Foundation under Contract $\Phi-809$.

[1] S. Frullani and J. Mougey, Adv. Nucl. Phys. 14, 1 (1984).

[2] S. Boffi, C. Giusti, F.D. Pacati, and M. Radici, Electromagnetic Response of Atomic Nuclei, Oxford Studies in Nuclear Physics (Clarendon Press, Oxford, 1996).

[3] L. Lapikás, Nucl. Phys. A553, 297c (1993).

[4] M. Leuschner, J.R. Calarco, F.W. Hersman, E. Jans, G.J. Kramer, L. Lapikás, G. van der Steenhoven, P.K.A. de Witt Huberts, H.P. Blok, N. Kalantar-Nayestanaki, and J. Friedrich, Phys. Rev. C 49, 955 (1994).

[5] J. Kramer, Ph.D. thesis, Amsterdam (1990).

[6] G. van der Steenhoven, H.P. Blok, E. Jeans, M. de Jong, L. Lapikás, E.N.M. Quint, and P.K.A. de Witt Huberts, Nucl. Phys. A480, 547 (1988).

[7] J.W.A. den Herder, H.P. Blok, E. Jeans, P.H.M. Keizer, L. Lapikás, E.N.M. Quint, G. van der Steenhoven, and P.K.A. de Witt Huberts, Nucl.Phys. A490, 507 (1988).

[8] A.N. Antonov, P.E. Hodgson and I.Zh. Petkov, Nucleon Momentum and Density Distributions in Nuclei (Clarendon Press, Oxford, 1988).

[9] A.N. Antonov, P.E. Hodgson and I.Zh. Petkov, Nucleon Correlations in Nuclei (Springer-Verlag, Berlin, 1993).

[10] C. Ciofi degli Atti, E. Pace, and G. Salmè, Phys. Rev. C 43, 1155 (1991). 
[11] C. Giusti and F.D. Pacati, Nucl. Phys. A473, 717 (1987); Nucl. Phys. A485, 461 (1988).

[12] Y. Jin, D.S. Onley, and L.E. Wright, Phys. Rev. C 45, 1311 (1992);

Y. Jin, J.K. Zhang, D.S. Onley, and L.E. Wright, Phys. Rev. C 47, 2024 (1993);

Y. Jin and D.S. Onley, Phys. Rev. C 50, 377 (1994);

J.M. Udías, P. Sarriguren, E. Moya de Guerra, E. Garrido, and J.A. Caballero, Phys. Rev. C 48, 2731 (1993).

[13] L. Lapikás, J. Wesseling, and R.B. Wiringa, nucl-th/9904008.

[14] A. Polls, M. Radici, S. Boffi, W.H. Dickhoff, and H. Müther, Phys. Rev. C 55, 810 (1997).

[15] K. Amir-Azimi-Nili, H. Müther, L.D. Skouras, and A. Polls, Nucl. Phys. A604, 245 (1996);

K. Amir-Azimi-Nili, J.M. Udías, H. Müther, L.D. Skouras, and A. Polls, Nucl. Phys. A625, 633 (1997).

[16] W.J.W. Geurts, K. Allaart, W.H. Dickhoff, and H. Müther, Phys. Rev. C 53, 2207 (1996).

[17] D. Van Neck, M. Waroquier, and K. Heyde, Phys. Lett. B 314, 255 (1993).

[18] M.V. Stoitsov, S.S. Dimitrova, and A.N. Antonov, Phys. Rev. C 53, 1254 (1996).

[19] D. Van Neck, L. Van Daele, Y. Dewulf, and M. Waroquier, Phys. Rev. C 56, 1398 (1997).

[20] S.S. Dimitrova, M.K. Gaidarov, A.N. Antonov, M.V. Stoitsov, P.E. Hodgson, V.K. Lukyanov, E.V. Zemlyanaya, and G.Z. Krumova, J. Phys. G 23, 1685 (1997).

[21] M.K. Gaidarov, K.A. Pavlova, S.S. Dimitrova, M.V. Stoitsov, and A.N. Antonov, Proceedings of XIV Intern. Seminar on High Energy Physics Problems, Dubna (Russia), 1998, in print.

[22] M.K. Gaidarov, K.A. Pavlova, S.S. Dimitrova, M.V. Stoitsov, A.N. Antonov, D. Van Neck, and H. Müther, nucl-th/9904022, Phys. Rev. C. 60 No.1 (1999), in print.

[23] M.V. Stoitsov, A.N. Antonov, and S.S. Dimitrova, Phys. Rev. C 47, R455 (1993); Phys. Rev. C 48, 74 (1993); Z. Phys. A 345, 359 (1993).

[24] F. Arias de Saavedra, G. Co', A. Fabrocini, and S. Fantoni, Nucl. Phys. A605, 359 (1996).

[25] A. Polls, H. Müther, and W.H. Dickhoff, Proceedings of Conference on Perspectives in Nuclear Physics at Intermediate Energies, Trieste, 1995, edited by S. Boffi, C. Ciofi degli Atti, and M.M. Giannini, (World Scientific, Singapore, 1996), p.308.

[26] G. Benenti, C. Giusti, and F.D. Pacati, Nucl. Phys. A574, 716 (1994). 
[27] S. Boffi, F. Cannata, F. Capuzzi, C. Giusti, and F.D. Pacati, Nucl. Phys. A379, 509 (1982).

[28] S. Boffi and M. Radici, Nucl. Phys. A526, 602 (1991).

[29] V. Van der Sluys, J. Ryckebusch, and M. Waroquier, Phys. Rev. C 49, 2695 (1994).

[30] J.E. Amaro, A.M. Lallena, and J. A. Caballero, nucl-th/9904030.

[31] F.G. Perey, In Direct Interactions and Nuclear Reactions Methods, eds. E. Clementel and C. Villi (Gordon and Breach, New York 1963), p.125;

F. Capuzzi, In The Nuclear Optical Model Potential, eds. S. Boffi and G. Passatore, Lecture Notes in Physics 55, 20 (1976).

[32] T. de Forest, Jr., Nucl. Phys. A392, 232 (1983).

[33] C. Mahaux and R. Sartor, Adv. Nucl. Phys. 20, 1 (1991).

[34] T. Berggren, Nucl. Phys. 72, 337 (1965).

[35] J.M. Bang, F.A. Gareev, W.T. Pinkston, and J.S. Vaagen, Phys. Rep. 125, 253 (1985).

[36] D. Van Neck, A.E.L. Dieperink, and M. Waroquier, Z. Phys. A 355, 107 (1996); Phys. Rev. C 53, 2231 (1996).

[37] S. Boffi, R. Cenni, C. Giusti, and F.D. Pacati, Nucl. Phys. A420, 38 (1984);

S. Boffi, F. Capuzzi, C. Giusti, and F.D. Pacati, Nucl. Phys. A436, 438 (1985).

[38] S. Boffi, C. Giusti, and F.D. Pacati, Nucl. Phys. A358, 91 (1981).

[39] J.I Johansson, H.S. Sherif, and G.M.Lotz, Nucl. Phys. A605, 517 (1996);

J.I Johansson and H.S. Sherif, Phys. Rev. C 56, 328 (1997).

[40] M. Gari and H. Hebach, Phys. Rep. 72, 1 (1981);

M. Cavinato, M. Marangoni, and A.M. Saruis, Nucl. Phys. A422, 237 (1984);

J. Ryckebusch, M. Waroquier, K. Heyde, and D. Ryckbosch, Phys. Lett. B 194, 453 (1987);

J. Ryckebusch, M. Waroquier, K. Heyde, J. Moreau, and D. Ryckbosch, Nucl. Phys. A476, 237 (1988);

J. Ryckebusch, K. Heyde, D. Van Neck, and M. Waroquier, Phys. Lett. B 216, 252 (1989);

J.P. McDermott, E. Rost, J.R. Shepard, and C.Y. Cheung, Phys. Rev. Lett. 61, 814 (1988);

D.G. Ireland and G. van der Steenhoven, Phys. Rev. C 49, 2182 (1994);

E.C. Aschenauer, I. Bobeldijk, D.G. Ireland, L. Lapikás, D. Van Neck, B. Schröder, V. Van der Sluys, G. 
van der Steenhoven, and R.E. Van de Vyver, Phys. Lett. B 389, 470 (1996);

L.J. de Bever, D. Branford, J.-O. Adler, B.-E. Andersson, I Bobeldijk, T. Davinson, R.S. Hicks, D.G. Ireland, K. Livingston, R.L.J. van der Meer, R.A. Miskimen, D. Van Neck, L. Isaksson, R.D. Page, G.A. Peterson, J. Rahighi, H. Ruijter, B. Schröder, G. van der Steenhoven, P.J. Woods, K. Wang, and A.C. Shotter, Phys. Rev. C 58, 981 (1998).

[41] H. Müther and W.H. Dickhoff, Phys. Rev. C 49, R17 (1994);

H. Müther, A. Polls, and W.H. Dickhoff, Phys. Rev. C 51, 3051 (1995).

[42] C. Giusti, F.D. Pacati, K. Allaart, W.J.W. Geurts, W.H. Dickhoff, and H. Müther, Phys. Rev. C 57, 1691 (1998);

C.J.G. Onderwater, K. Allaart, E.C. Aschenauer, Th.S. Bauer, D.J. Boersma, E. Cisbani, W.H. Dickhoff, S. Frullani, F. Garibaldi, W.J.W. Geurts, C. Giusti, D. Groep, W.H.A. Hesselink, M. Iodice, E. Jans, N. Kalantar-Nayestanaki, W.-J. Kasdorp, C. Kormanyos, L. Lapikás, J.J. van Leeuwe, R. De Leo, A. Misiejuk, H. Müther, F.D. Pacati, A.R. Pellegrino, R. Perrino, R. Starink, M. Steenbakkers, G. van der Steenhoven, J.J.M. Steijger, M.A. van Uden, G.M. Urciuoli, L.B. Weinstein, and H.W. Willering, Phys. Rev. Lett. 81, $2213(1998)$.

[43] P. Schwandt, H.O. Meyer, W.W. Jacobs, A.D. Bacher, S.E. Vigdor, M.D. Kaitchuck, and T.R. Donoghue, Phys. Rev. C 26, 55 (1982).

[44] S. Boffi, C. Giusti, F.D. Pacati, and F. Cannata, Nuovo Cim. 98, 291 (1987).

[45] D. Van Neck, M. Waroquier, A.E.I. Dieperink, S.C. Pieper, and V.R. Pandharipande, Phys. Rev. C 57, 2308 (1998).

[46] D.J.S. Findlay and R.O. Owens, Nucl. Phys. A279, 389 (1977).

[47] F. de Smet, H. Ferdinande, R. Van de Vyver, L. Van Hoorebeke, D. Ryckbosch, C. Van den Abeele, J. Dias, and J. Ryckebusch, Phys. Rev. C 47, 652 (1993).

[48] G.J. Miller, J.C. McGeorge, J,R.M. Annand, G.I. Crawford, V. Holliday, I.J.D. MacGregor, R.O. Owens, J. Ryckebusch, J.-O. Adker, B.-E. Andersson, L. Isaksson, and B. Schröder, Nucl. Phys. A586, 125 (1995). 
Table 1: Spectroscopic factors for the ${ }^{16} \mathrm{O}\left(e, e^{\prime} p\right)$ knockout reaction leading to the $1 / 2^{-}$ground state and to the $3 / 2^{-}$excited state of ${ }^{15} \mathrm{~N}$. Column I gives the spectroscopic factors deduced from the calculations with different $\mathrm{OBDM}$ of ${ }^{16} \mathrm{O}$; II gives the additional reduction factors determined through a comparison between the $\left(e, e^{\prime} p\right)$ data of ref. [- $\mid$ and the reduced cross sections calculated in DWIA with the different overlap functions; III gives the total spectroscopic factors obtained from the product of the factors in columns I and II.

\begin{tabular}{|c|c|c|c|c|c|c|}
\hline \multirow[b]{2}{*}{ OBDM } & \multicolumn{3}{|c|}{$1 p_{1 / 2}$} & \multicolumn{3}{|c|}{$1 p_{3 / 2}$} \\
\hline & I & II & III & I & II & III \\
\hline $\mathrm{HF}$ & 1.000 & 0.750 & 0.750 & 1.000 & 0.550 & 0.550 \\
\hline JCM 18 & 0.953 & 0.825 & 0.786 & 0.953 & 0.600 & 0.572 \\
\hline CBF 19 & 0.912 & 0.850 & 0.775 & 0.909 & 0.780 & 0.709 \\
\hline $\mathrm{CBF} 24$ & 0.981 & 0.900 & 0.883 & 0.981 & 0.600 & 0.589 \\
\hline GFM 25 & 0.905 & 0.800 & 0.724 & 0.915 & 0.625 & 0.572 \\
\hline
\end{tabular}


FIG. 1. Reduced cross section of the ${ }^{16} \mathrm{O}\left(e, e^{\prime} n\right)$ reaction as a function of the missing momentum $p_{\mathrm{m}}$ for the transitions to the $1 / 2^{-}$ground state and the first $3 / 2^{-}$excited state of ${ }^{15} \mathrm{O}$ in parallel kinematics, with $E_{0}=520.6$ $\mathrm{MeV}$ and an outgoing neutron energy of $100 \mathrm{MeV}$. The optical potential is from ref. 443. Overlap functions derived from the OBDM of GFM 25] (solid lines), CBF 19] (dashed lines), CBF 24] (dot-dashed lines), JCM [18] (double dot-dashed lines). The dotted lines are calculated with the HF wave function. The positive (negative) values of $p_{\mathrm{m}}$ refer to situations where $|\boldsymbol{q}|<\left|\boldsymbol{p}^{\prime}\right|\left(|\boldsymbol{q}|>\left|\boldsymbol{p}^{\prime}\right|\right)$.

FIG. 2. Reduced cross section of the ${ }^{16} \mathrm{O}\left(e, e^{\prime} p\right)$ reaction as a function of the missing momentum $p_{\mathrm{m}}$ for the transitions to the $1 / 2^{-}$ground state and the first $3 / 2^{-}$excited state of ${ }^{15} \mathrm{~N}$ in parallel kinematics, with $E_{0}=520.6$ $\mathrm{MeV}$ and an outgoing proton energy of $90 \mathrm{MeV}$. The optical potential is from ref. [43] (see Table III of ref. [4]). Line convention is as in Fig. 1. The experimental data are taken from ref. A. The theoretical results have been multiplied by the reduction factor given in column II of Table I.

FIG. 3. Angular distribution of the cross section of the ${ }^{16} \mathrm{O}(\gamma, p)$ reaction for the transition to the $1 / 2^{-}$ground state of ${ }^{15} \mathrm{~N}$ at $E_{\gamma}=60 \mathrm{MeV}$. The separate contribution given by the one-body current (DWIA) and the final result given by the sum of the one-body and the two-body seagull current (DWIA+MEC) are shown. Line convention is as in Fig. 1. The optical potential is from ref. 443. The experimental data are taken from ref. 46. (black circles), ref. 47 (open circles) and ref. 48 (triangles). The theoretical results have been multiplied by the reduction factors listed in column II of Table I, consistently with the analysis of $\left(e, e^{\prime} p\right)$ data.

FIG. 4. Angular distribution of the cross section of the ${ }^{16} \mathrm{O}(\gamma, p)$ reaction for the transition to the $1 / 2^{-}$ground state of ${ }^{15} \mathrm{~N}$ at $E_{\gamma}=72 \mathrm{MeV}$. The separate contribution given by the one-body current (DWIA) and the final result given by the sum of the one-body and the two-body seagull current (DWIA+MEC) are shown. Line convention is as in Fig. 3. The optical potential is from ref. 43. The experimental data are taken from ref. 448. The theoretical results have been multiplied by the reduction factors listed in column II of Table I, consistently with the analysis of $\left(e, e^{\prime} p\right)$ data.

FIG. 5. Angular distribution of the cross section of the ${ }^{16} \mathrm{O}(\gamma, p)$ reaction for the transition to the $3 / 2^{-}$state of ${ }^{15} \mathrm{~N}$ at $6.3 \mathrm{MeV}$ and at $E_{\gamma}=72 \mathrm{MeV}$. The separate contribution given by the one-body current (DWIA) and the final result given by the sum of the one-body and the two-body seagull current (DWIA+MEC) are shown. Line convention and the optical potential are as in Fig. 4. The experimental data are taken from ref. 48. The theoretical results have been multiplied by the reduction factors listed in column II of Table I, consistently with the analysis of $\left(e, e^{\prime} p\right)$ data. 


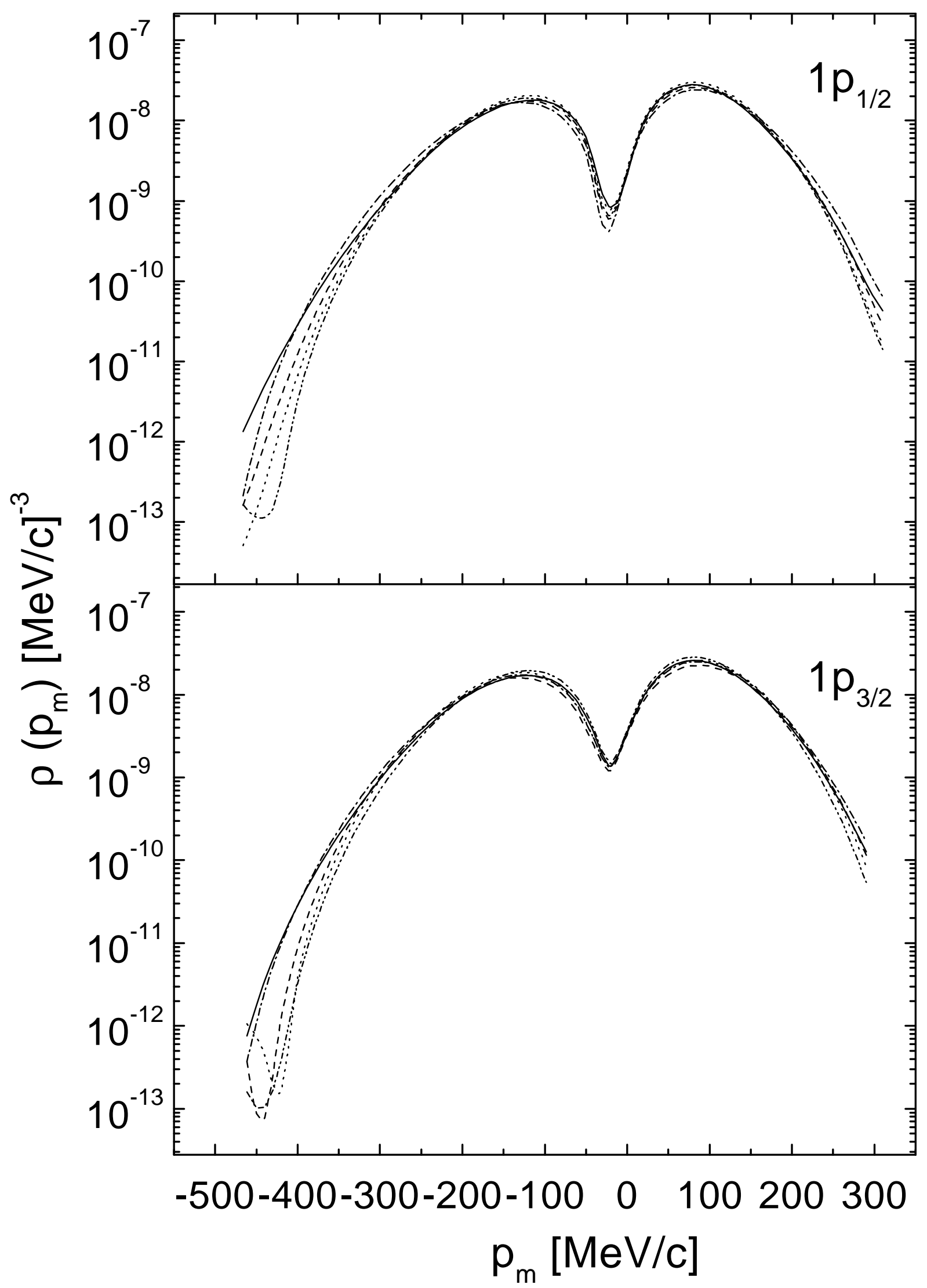




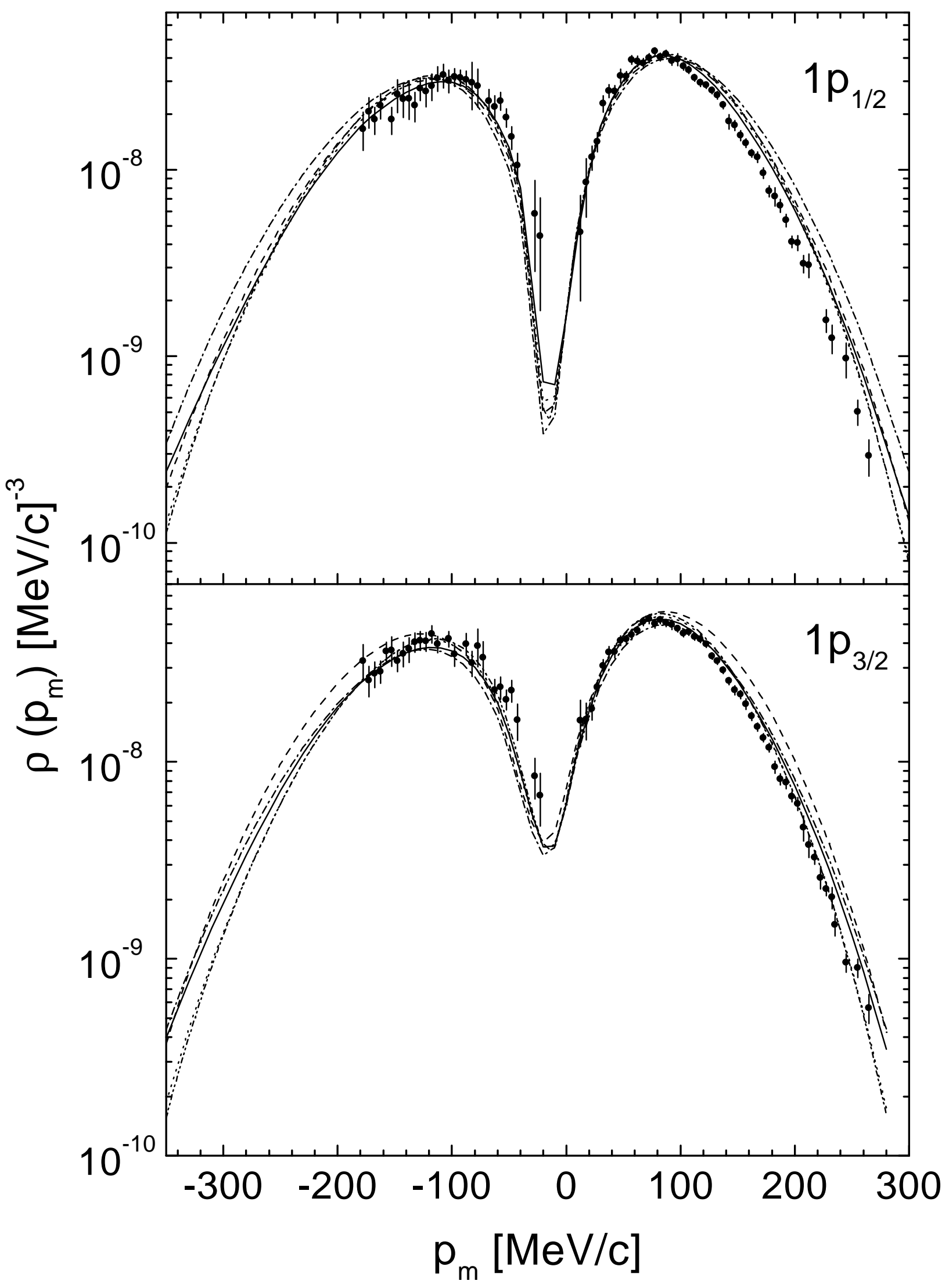




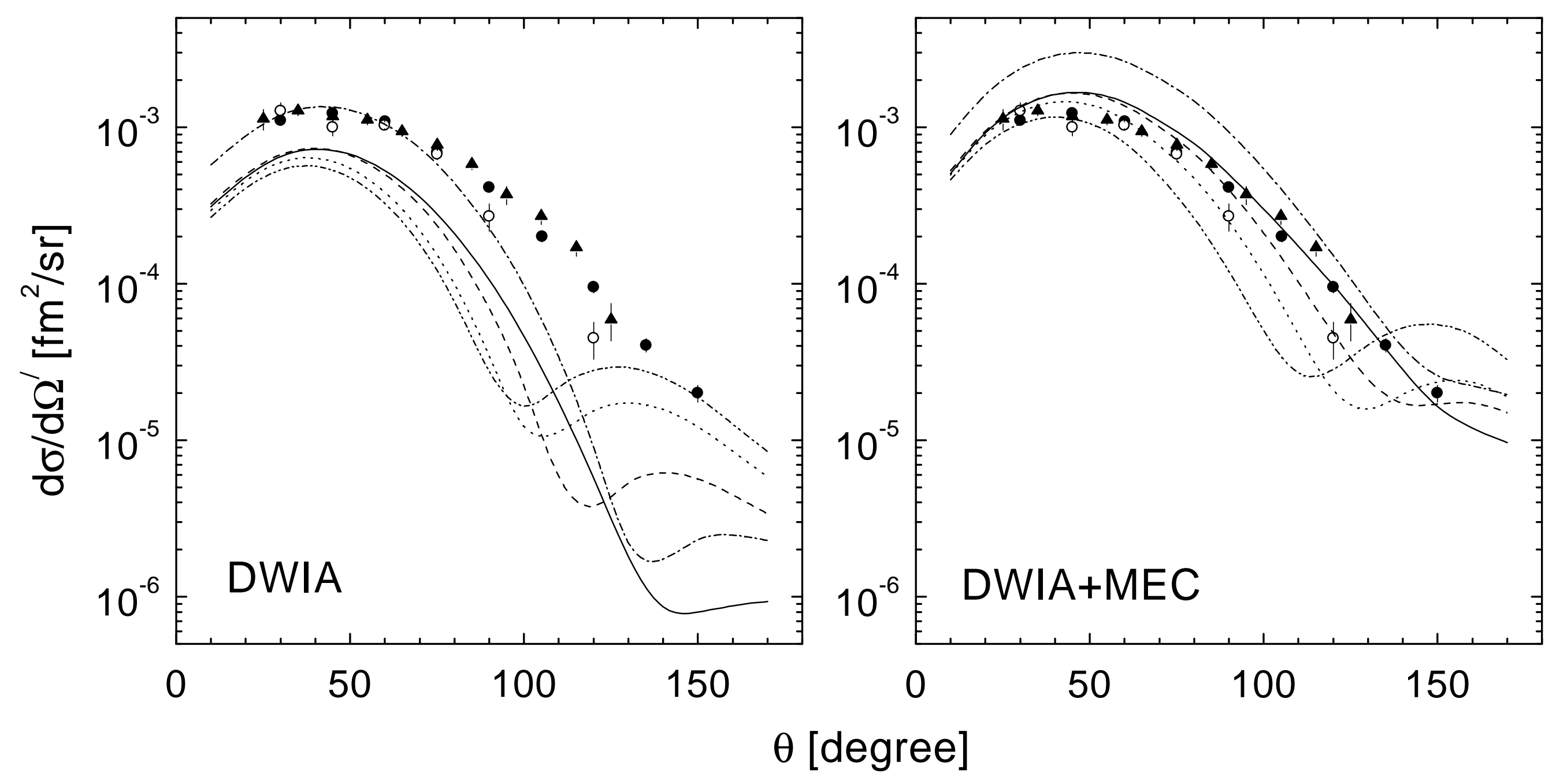




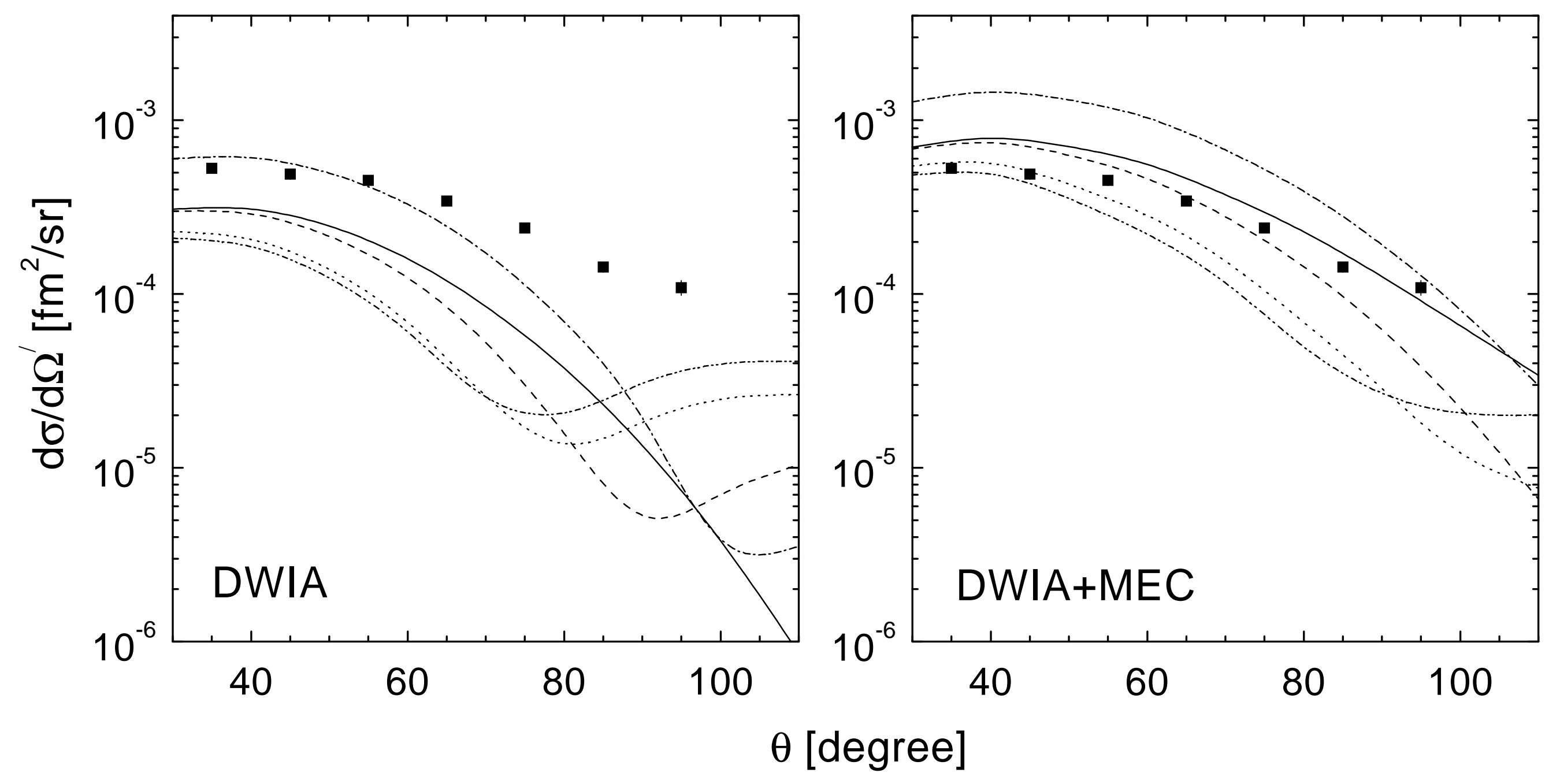




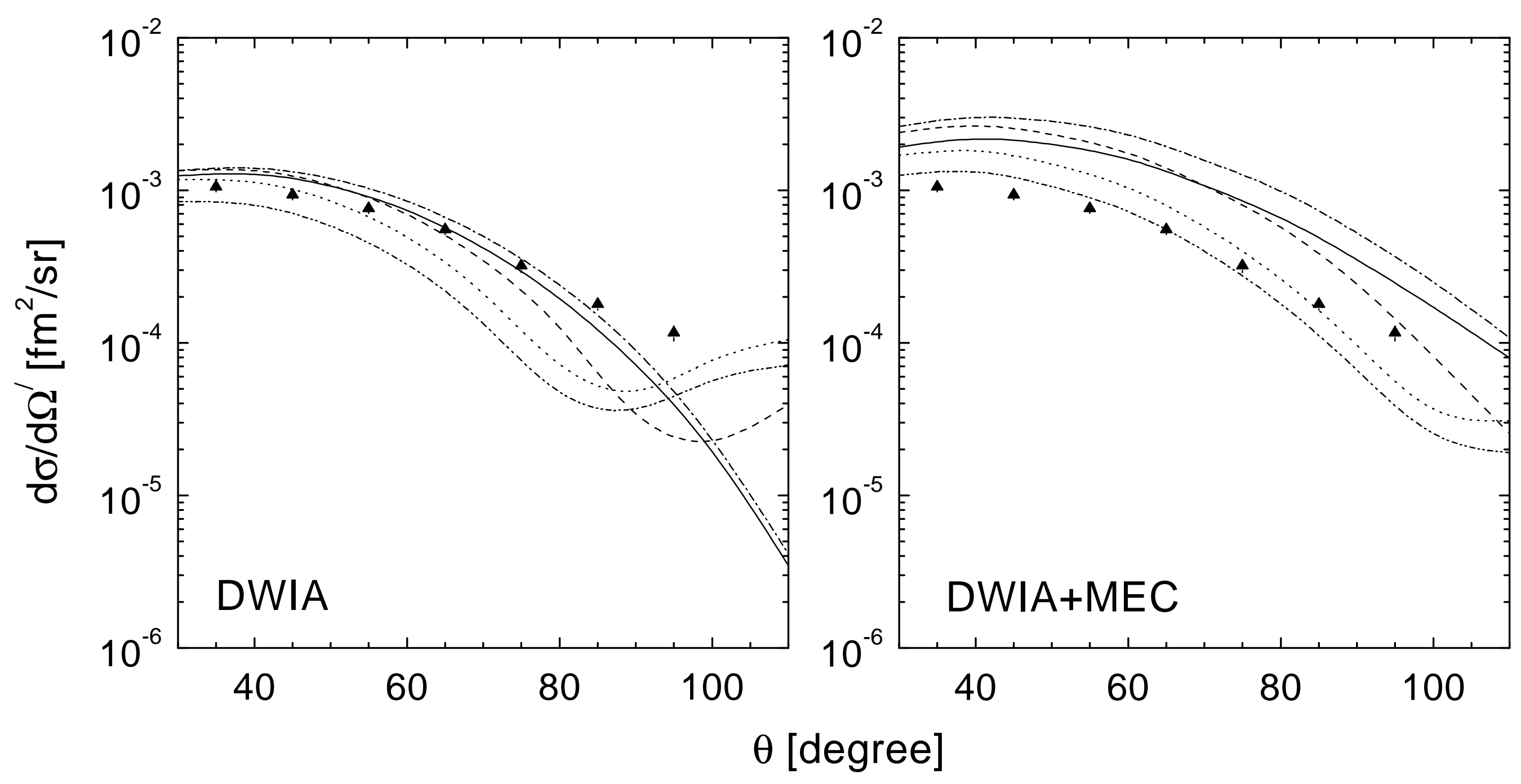

